\title{
Combined Heat Transfer in High-Porosity High- Temperature Fibrous Insulations: Theory and Experimental Validation
}

\author{
Kamran Daryabeigi* \\ NASA Langley Research Center, Hampton, Virginia, 23681 \\ George R. Cunnington ${ }^{\dagger}$ \\ Cunnington and Associates, Palo Alto, California, 94303 \\ Steve D. Miller \\ S.D. Miller \& Associates Research Foundation, Flagstaff, AZ, 86004 \\ and \\ Jeffry R. Knutson ${ }^{\S}$ \\ NASA Langley Research Center, Hampton, Virginia, 23681
}

\begin{abstract}
Combined radiation and conduction heat transfer through various high-temperature, high-porosity, unbonded (loose) fibrous insulations was modeled based on first principles. The diffusion approximation was used for modeling the radiation component of heat transfer in the optically thick insulations. The relevant parameters needed for the heat transfer model were derived from experimental data. Semi-empirical formulations were used to model the solid conduction contribution of heat transfer in fibrous insulations with the relevant parameters inferred from thermal conductivity measurements at cryogenic temperatures in a vacuum. The specific extinction coefficient for radiation heat transfer was obtained from high-temperature steady-state thermal measurements with large temperature gradients maintained across the sample thickness in a vacuum. Standard gas conduction modeling was used in the heat transfer formulation. This heat transfer modeling methodology was applied to silica, two types of alumina, and a zirconia-based fibrous insulation, and to a variation of opacified fibrous insulation (OFI). OFI is a class of insulations manufactured by embedding efficient ceramic opacifiers in various unbonded fibrous insulations to significantly attenuate the radiation component of heat transfer. The heat transfer modeling methodology was validated by comparison with more rigorous analytical solutions and with standard thermal conductivity measurements. The validated heat transfer model is applicable to various densities of these high-porosity insulations as long as the fiber properties are the same (index of refraction, size distribution, orientation, and length). Furthermore, the heat transfer data for these insulations can be obtained at any static pressure in any working gas environment without the need to perform tests in various gases at various pressures.
\end{abstract}

\section{Nomenclature}

$c \quad=$ specific heat, $\mathrm{J} /(\mathrm{kg} . \mathrm{K})$

$d_{p} \quad=$ fiber diameter, $\mathrm{m}$

$d_{m} \quad=$ gas collision diameter, $\mathrm{m}$

\footnotetext{
* Aerospace Engineer, Structural Mechanics and Concepts Branch, Mail Stop 190. Senior Member, AIAA.

${ }^{\dagger}$ Owner, Cunnington and Associates. Associate Fellow, AIAA.

* Manager, S.D. Miller \& Associates Research Foundation/Congressional District Programs.

$\S$ Electrical Engineering Technician, Systems Integration and Test Branch, Mail Stop 424.
}

1

American Institute of Aeronautics and Astronautics 

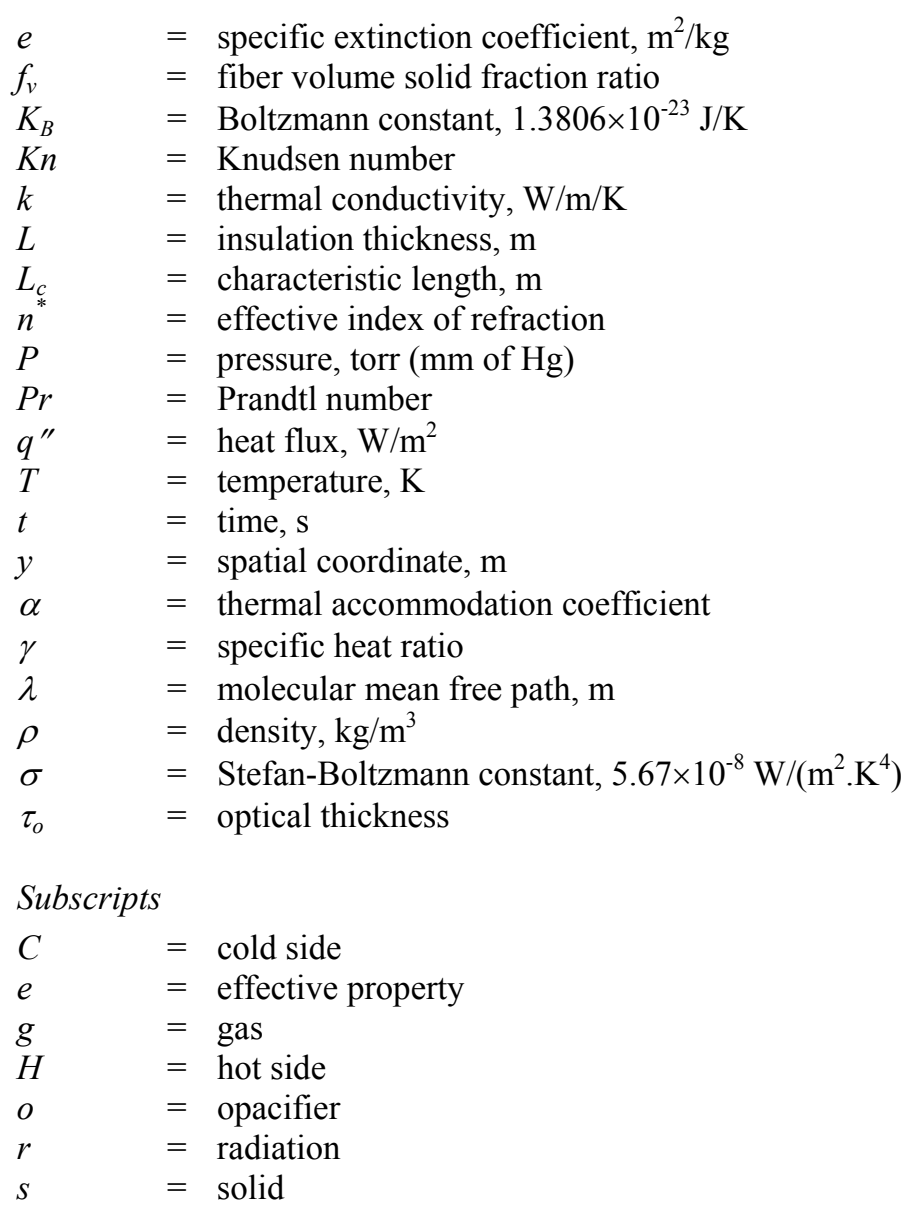

\section{Introduction}

Weat transfer through fibrous insulations has been the subject of great interest in the aerospace community because of their use in thermal protection systems (TPS) for moderate to high temperature applications. The fibrous insulation systems used are either in bonded or unbonded form. The unbonded form consists of loose fibrous insulation mats typically used in blankets, while the bonded form is manufactured by sintering fibrous insulation mats to obtain a rigid insulation. The advanced flexible reusable surface insulation (AFRSI) blankets and the rigidized reusable surface insulation tiles on the Space Shuttle Orbiter are examples of unbonded and bonded fibrous insulations, respectively. The focus of this paper is on unbonded fibrous insulations even though the basic heat transfer formulation applies to both insulations of various compositions.

Heat transfer through these high-porosity insulations is composed of combined radiation and conduction heat transfer. Conduction consists of both solid and gaseous conduction. In fibrous insulations with densities of $20 \mathrm{~kg} / \mathrm{m}^{3}$ or higher, natural convection is insignificant. ${ }^{1,2}$ Forced convection in fibrous insulations is insignificant in the absence of sufficient pressure gradient and flow path across the insulation. Solid conduction is the least significant component of heat transfer in high-porosity, unbonded fibrous insulations. Radiation and gas conduction are the dominant modes of heat transfer. Radiation's significance increases with increasing temperature, and decreases with increasing insulation density. Gas conduction is negligible in a vacuum, and increases with increasing static pressure and temperature.

The standard practice for modeling heat transfer through fibrous insulations uses thermal conductivity measurements as a function of temperature and pressure obtained using either steady-state ${ }^{3}$ or transient $^{4}$ techniques. The tabulated thermal conductivity data are then used for analysis and design of TPS. One shortcoming of this technique is that the generated thermal conductivity data are applicable only to the specific composition and gaseous test environment; if the density or gaseous environment is changed, a new set of data is needed. Furthermore, the measured thermal conductivity lumps the contributions of the various modes of heat transfer without providing any insight into the physics or the contribution of each heat transfer mode. The advantage of the physics-based modeling 
presented here is to provide insight into the significance of various modes of heat transfer through the insulation at various temperatures and pressures, which can then be used for optimizing insulation designs. Furthermore, once the solid conduction contribution and extinction coefficient are known from measurements under vacuum conditions, thermal data may be generated for any insulation density at any pressure and temperature in any gaseous environment as long as the fiber properties are the same (index of refraction, size distribution, orientation, length).

Theoretical modeling of solid conduction through fibers and points of contact between them is difficult, therefore, various empirical and semi-empirical relations have traditionally been used. ${ }^{5,}{ }^{6}$ Modeling of gas conduction in fibrous insulations requires knowledge of characteristic length (pore size), and gas mean free path, and is well established. Modeling of radiation heat transfer through fibrous insulations is more complicated and has been the subject of numerous studies, with a comprehensive review of various radiation models provided by Lee and Cunnington. ${ }^{8}$ Significant work has been devoted to developing physics-based heat transfer modeling for bonded and unbonded fibrous insulations with various degrees of complexity. The simplest approach has been to use a combined radiation and conduction heat transfer model with radiation conductivity in terms of a Rosseland mean coefficient based on the diffusion approximation. ${ }^{9,}{ }^{10}$ Later models used more refined radiation models based on scattering intensity distribution from infinite cylinders from the solution of Maxwell's equations. ${ }^{11,}{ }^{12}$ These more complex radiation models did not yield better agreement with experimental data, because they did not properly account for the two-dimensional scattering characteristics of fibers in the medium. Lee developed a rigorous formulation for scattering properties of fibrous insulations that accounted for the two-dimensional scattering characteristics of fibers, with the resulting radiation model using deterministic parameters that define the composition and morphology of the medium: distributions of fiber size and orientation, fiber volume fraction, and the spectral complex refractive index of the fibers. ${ }^{13,14}$ This radiation model is limited to the independent scattering regime, requiring detailed knowledge of fiber orientation with respect to the boundaries, and accurate spectral complex refractive index data. Up to the present, this formulation has only been successfully developed and validated for a silica fiber and a soda-lime glass fiber insulation. ${ }^{6}$

The objective of the present work was to investigate whether simple thermal test data may be used to accurately estimate relevant parameters needed for combined radiation and conduction heat transfer in various optically thick unbonded fibrous insulations. The methodology consists of estimation of a semi-empirical solid conduction model from thermal measurements in a vacuum at cryogenic temperatures, and estimation of the specific extinction coefficient from steady-state test data in a vacuum at various temperatures between 300 and $1400 \mathrm{~K}$. The standard gas conduction formulation is then applied to model heat transfer at higher pressures. This methodology was applied to silica $\left(\mathrm{Q}-\mathrm{Fiber}^{* *}\right)$, alumina [Saffil ${ }^{\dagger \dagger}$ and alumina paper $\left(\mathrm{APA}^{\$}\right)$ ], zirconia $\left(\mathrm{ZYF}^{\S \S}\right)$, and $\mathrm{OFI}^{* * *}$ insulations. Validation of the methodology consisted of comparing the experimentally estimated specific extinction coefficient of Q-Fiber with the theoretical formulation of Lee and Cunnington, ${ }^{6}$ and comparing estimated thermal conductivity with standard thermal conductivity measurements of Saffil and APA.

\section{Analytical Approach}

In the absence of forced or natural convection, the governing one-dimensional conservation of energy equation for the problem of combined radiation and conduction in a radiation participating medium is given by

$$
\rho c \frac{\partial T}{\partial t}=\frac{\partial}{\partial y}\left(k_{c} \frac{\partial T}{\partial y}\right)-\frac{\partial q_{r}^{\prime \prime}}{\partial y}
$$

where $k_{c}$ is combined gas/solid thermal conductivity. For an optically thick medium, the diffusion approximation can be used resulting in the radiant heat flux of

$$
q_{r}^{\prime \prime}=-k_{r} \frac{\partial T}{\partial y}
$$

Using this approximation Eq. (1) reduces to

$$
\rho c \frac{\partial T}{\partial t}=\frac{\partial}{\partial y}\left(k \frac{\partial T}{\partial y}\right)
$$

\footnotetext{
** Johns Manville Corporation

${ }^{\dagger}$ Thermal Ceramics Inc

\# Zircar Ceramics Inc

$\S \S$ Zircar Zirconia Inc

${ }^{* * *}$ S. D. Miller \& Associates Research Foundation/CDP
}

American Institute of Aeronautics and Astronautics 
where $k$ is obtained by superposition of the thermal conductivities due to solid conduction, gas conduction, and radiation

$$
k=k_{s}+k_{g}+k_{r}
$$

The formulation for each of the thermal conductivity terms is discussed in detail.

\section{A. Radiation}

The radiant thermal conductivity for regular and opacified fibrous insulations is provided by Eqs. (5a) and (5b), respectively

$$
\begin{gathered}
k_{r}=\frac{16 \sigma{ }^{* 2} T^{3}}{3 \rho e} \\
k_{r}=\frac{16 \sigma T^{3}}{3\left(\frac{\rho e}{n^{* 2}}+\frac{\rho_{o} e_{o}}{n_{o}^{* 2}}\right)}
\end{gathered}
$$

The first and second quotients in the denominator of Eq. (5b) contain fiber and opacifier properties, respectively. Specific extinction coefficient is an intrinsic property of fibers and is a function of temperature, fiber material, fiber diameter, and fiber orientation. Typically, the extinction coefficient is a nonlinear function of temperature. The optical thickness, $\tau_{0}$, is defined as

$$
\tau_{0}=\rho e L
$$

Insulation can be considered optically thick only if $\tau>>1$, which is valid for insulation samples used in this study with optical thicknesses $>20$.

The effective index of refraction of fibrous media and opacifiers is a strong function of fiber/opacifier volume fraction $\left(f_{v}\right)$ and fiber properties, ${ }^{15}$ and a weak function of temperature. The temperature dependence was neglected and a curve fit of the effective index of refraction with fiber/opacifier volume fraction was used, with the effective index of refraction increasing with increasing fiber/opacifier volume fraction. The effective index of refractions of silica, alumina, and ceramic opacifier were obtained from the index of refraction of these materials based on modeling by Caren, ${ }^{15}$ and typically varied between 1.01 and 1.09 , while the effective index of refraction of zirconia was not known. The specific extinction coefficients for the silica and alumina fibrous insulations and the ceramic opacifier used in OFI were estimated from the experimental data using a genetic algorithm-based parameterestimation technique. ${ }^{16}$ For zirconia, the combined quantity $e / n^{* 2}$ was estimated.

\section{B. Solid Conduction}

An exact theoretical formulation of solid conduction for the fibrous insulation geometry is a formidable task because of the tenuous nature of the myriad of different paths of varying fiber lengths and cross sectional areas. A semi-empirical approach was used to model solid conduction heat transfer in unbonded fibers

$$
k_{S}(T)=F_{s} f_{v}^{b} k_{S}^{*}(T)
$$

which relates the solid thermal conductivity of fibrous insulation to the thermal conductivity of bulk fiber material, $k_{s}{ }^{*}$, and fiber volume fraction. The model assumes that the solid conductivity is independent of fiber diameter and varies with fiber volume fraction raised to a power, ${ }^{5,17}$ typically between 1 and 3 . The parameter $F_{s}$ is a global property that relates the micro-scale geometric effects of fiber matrix with bulk dimensions, and accounts for the various fiber path lengths, fiber arrangement, and fiber to fiber contacts, and is assumed to be temperature independent. $F_{s}$ and $b$ were obtained from thermal conductivity measurements in a vacuum at cryogenic temperatures (test condition with negligible gas conduction and reduced radiation).

\section{Gas Conduction}

Gas thermal conductivity does not vary with pressure, but the exchange of heat from gas molecules to adjacent solid surfaces is influenced by the environmental pressure in the rarefied and transition flow transport regimes. Thus, an effective gas thermal conductivity was defined as ${ }^{7}$ 


$$
k_{g}(T, P)=\frac{k_{g 0}(T)}{\Phi+2 \Psi \frac{\beta}{\operatorname{Pr}} K n}
$$

where $k_{g 0}(T)$ is the thermal conductivity of the gas. The parameter $\beta$ is defined as

$$
\beta=\left(\frac{2-\alpha}{\alpha}\right) \frac{2 \gamma}{(\gamma+1)}
$$

Since there is limited data for thermal accommodation coefficient between various gases and fiber materials, a thermal accommodation coefficient of unity was used for all the fibrous insulations and gaseous environments considered in the present study. Parameters $\Phi$ and $\Psi$ depend on the Knudsen number. $\Phi=1, \Psi=0$ for $\mathrm{K}_{\mathrm{n}}<0.01$ (continuum regime), $\Phi=1, \Psi=1$ for $0.01<\mathrm{K}_{\mathrm{n}}<10$ (transition regime), and $\Phi=0, \Psi=1$ for $\mathrm{Kn}>10$ (freemolecular regime). The Knudsen number is defined as

$$
K n=\frac{\lambda}{L_{c}}
$$

while the gas mean free path is

$$
\lambda=\frac{K_{B} T}{\sqrt{2} \pi d_{m}^{2} P}
$$

Once $k_{g o}(T), \operatorname{Pr}(T), \gamma(T)$, and $d_{m}$ are known from literature, the gas conduction contribution in fibrous insulations can be easily calculated if the gas conduction characteristic length (pore size) is known. The pore size, $L_{c}$, is not easy to define, therefore, an empirical formulation is typically used

$$
L_{c}=\xi \frac{d_{p}}{f_{v}}
$$

The constant $\xi$ is either 0.524 for fibers randomly oriented in space, ${ }^{18}$ or 0.785 for fibers randomly oriented in planes. ${ }^{5}$ The latter was used in this study because it produced better agreement with experimental results. A comparison between experimental measurements of pore diameters and calculated values for fibrous materials is given by Kaganer. ${ }^{19}$

The analytical thermal conductivity in Eq. (4) is obtained by substituting for radiant, solid, and gas thermal conductivity terms from Eqs. (5a) or (5b), (7), and (8), respectively.

\section{Experimental Approach}

Data reported here were generated using the steady-state thermal test setup at NASA Langley Research Center (LaRC) which provides effective thermal conductivity data with large temperature gradients maintained across the sample thickness. A limited set of thermal conductivity data was also generated using the transient radiant step heating technique mainly for validation of heat transfer models. The test samples and the measurement techniques are briefly described

\section{A. Test Samples}

Q-Fiber was the silica-based insulation used in this study. This is a well characterized insulation that is used in the AFRSI blankets. The mean fiber diameter is $1.3 \mu \mathrm{m}$. Effective thermal conductivity data at densities of 48.6, 68.8 , and $95.6 \mathrm{~kg} / \mathrm{m}^{3}$ with sample thicknesses of $13.3,19.1$, and $13.3 \mathrm{~mm}$, respectively, were used. The effective thermal conductivity test data on the samples with densities of 48.6 and $95.6 \mathrm{~kg} / \mathrm{m}^{3}$ had previously been reported elsewhere. ${ }^{20}$

Saffil with a mean fiber diameter of $4.5 \mu \mathrm{m}$ was one of the alumina-based fibrous insulations used in this study. Previously reported ${ }^{20}, 2$ effective thermal conductivity data on this insulation at various densities between 24.2 and $96.1 \mathrm{~kg} / \mathrm{m}^{3}$ for sample thicknesses varying between 13.3 and $39.3 \mathrm{~mm}$ were used in the present study. Radiation heat transfer in Saffil had previously been modeled using the modified two-flux approximation with isotropic ${ }^{21}$ and anisotropic ${ }^{2}$ scattering, for higher-order radiation modeling of thin layers of low density Saffil located between reflective foils in high-temperature multilayer insulations. ${ }^{21}$ 
APA with a mean fiber diameter of $4.5 \mu \mathrm{m}$ and a density of $107 \mathrm{~kg} / \mathrm{m}^{3}$ was the other alumina-based fibrous insulation sample used in this study. The APA insulation was provided in approximately 1-mm thick sheets, and 25.4-mm thick samples were used for the effective thermal conductivity measurements.

Yittria-stabilized zirconia (ZYF) fiber insulation with a mean fiber diameter of $6 \mu \mathrm{m}$ and a density of $267 \mathrm{~kg} / \mathrm{m}^{3}$ was used. The ZYF insulation was provided in approximately $2.5-\mathrm{mm}$ thick sheets, and a 25.4-mm thick sample was used for the effective thermal conductivity measurements.

OFI is a class of efficiently opacified fibrous insulation samples. The use of efficient ceramic opacifier particles embedded in fibrous insulation mats can significantly reduce the radiation component of heat transfer in fibrous insulations, thus making OFI an attractive insulation for low pressure applications such as sustained hypersonic flight or re-entry where most of the highest aeroheating occurs at altitudes with correspondingly low pressures. OFI samples have been produced by embedding the ceramic opacifiers in various fiber mats. The ratio of base fiber and ceramic opacifier can be tailored to provide an optimized insulation for the specific flight trajectory/areoheating load. The specific variation of OFI discussed in the present study was manufactured by embedding opacifiers in Saffil with an overall effective density of $202.4 \mathrm{~kg} / \mathrm{m}^{3}$. Effective thermal conductivity measurements were performed on a 25.4-mm thick sample.

\section{B. Effective Thermal Conductivity Measurements}

Steady-state effective thermal conductivity data with large temperature differences maintained across the sample thickness were generated in the LaRC thermal-vacuum testing apparatus, described in detail elsewhere. ${ }^{20,}{ }^{22} \mathrm{~A}$ schematic of the test setup is shown in Fig. 1. The main components are: a quartz lamp radiant heater array, an Inconel septum plate, a water-cooled plate, test sample, and refractory ceramic board insulation. The planar area of the water-cooled and septum plates is $304.8 \times 304.8 \mathrm{~mm}$. The insulation sample is typically $304.8 \times 304.8 \mathrm{~mm}$ by $25.4 \mathrm{~mm}$ thick and is placed between the water-cooled and septum plates. Use of a test sample with such a large ratio of planar dimension to thickness ensures one dimensional heat transfer in the test setup, especially in the central region of the test assembly. The water-cooled plate is equipped with nine flush-mounted thin-film heat flux gages that provide simultaneous heat flux and temperature measurements, while the septum plate is instrumented with 23 metal-sheathed Type K thermocouples. Data from instrumentation located in the central $127 \times 127 \mathrm{~mm}$ section of the test setup on the water-cooled and septum plates are used for the effective thermal conductivity measurements, while data from instrumentation located outside of the central region are used to assess deviations from one dimensional heat transfer in the setup. The average heat flux and temperature calculated from five heat flux gages located in the central region of the water-cooled plate provide the heat flux, q", and cold side temperature,

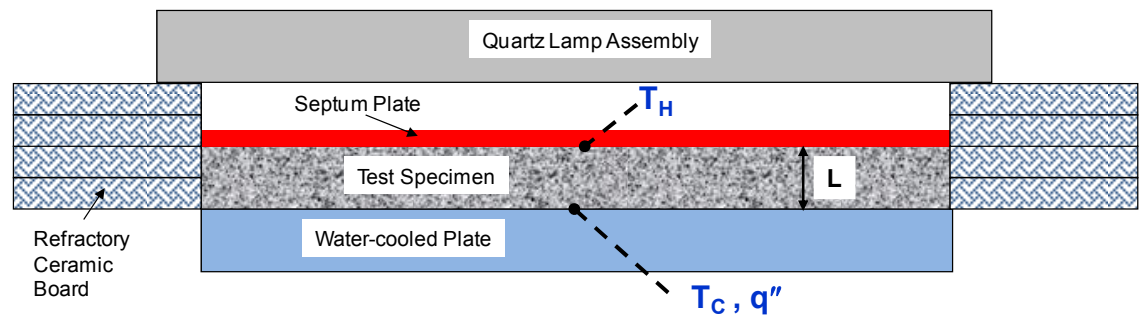

Figure 1. Schematic of steady-state test setup

$\mathrm{T}_{\mathrm{C}}$, while the average temperature calculated from 11 thermocouples located in the central region of the septum plate provides the hot side temperature, $\mathrm{T}_{\mathrm{H}}$. The effective thermal conductivity is then calculated from

$$
k_{e}=\frac{q^{\prime \prime} L}{\left(T_{H}-T_{C}\right)}
$$

For an optically thick insulation, the effective thermal conductivity is related to thermal conductivity through

$$
k_{e}=\frac{1}{T_{H}-T_{C}} \int_{T_{C}}^{T_{H}} k \partial T
$$

If thermal conductivity is a linear function of temperature, then the resulting effective thermal conductivity is the thermal conductivity calculated at the average temperature of $0.5\left(\mathrm{~T}_{\mathrm{H}}+\mathrm{T}_{\mathrm{C}}\right)$. But the thermal conductivity of the insulations studied here is a highly nonlinear function of temperature. Even though the test setup does not directly yield thermal conductivity, the measured effective thermal conductivities can be used in conjunction with the 
physics-based model of thermal conductivity according to the above equation to yield valuable data. It can be used to either validate heat transfer models, or estimate radiant/thermophysical properties needed in the heat transfer model. This test setup provides accurate, repeatable, one dimensional heat transfer data.

Tests were conducted with various $\mathrm{T}_{\mathrm{H}}$ between 530 and $1360 \mathrm{~K}$. At each $\mathrm{T}_{\mathrm{H}}$ set point, tests were conducted at various static pressures between 0.001 and 750 torr in nitrogen gas. The average $\mathrm{T}_{\mathrm{C}}$ for all the test data was $296.3 \pm$ $10 \mathrm{~K}$. A detailed uncertainty analysis ${ }^{23}$ was conducted for each measured quantity: hot and cold side temperatures, and heat fluxes. The overall uncertainty consisted of the contributions of random and bias uncertainties for each measured quantity, and uncertainties due to spatial non-uniformity of spatially-averaged quantities. The average experimental uncertainty for data reported here was $7 \%$ with root mean square (rms) deviation of $16.6 \%$. The highest uncertainties were at the lowest pressure and hot side temperature conditions (due to low signal to noise level associated with heat flux measurements at these conditions), and typically decreased with increasing pressure and hot side temperature.

For tests on ZYF and APA, four uncoated thin-foil thermocouples were installed at various heights through the sample thickness in the center of the test sample. Because these insulations are provided in thin sheets, it was easy to install the thermocouples between various sheets, and know the location of each thermocouple in the stack.

\section{Thermal Conductivity Measurements}

Standard thermal conductivity data were measured at a thermophysical properties laboratory using the transient radiant step heating technique referred to as the "three point method", described in detail elsewhere. ${ }^{24}$ This measurement technique was used to generate thermal conductivity data on Saffil and APA in the temperature range of $290-1173 \mathrm{~K}$ in a vacuum for validation of the heat transfer model. This measurement technique was also used on Saffil and ZYF for thermal conductivity measurements in a vacuum at cryogenic temperatures ( 130 to $270 \mathrm{~K}$ ), for inferring parameters needed for solid conduction modeling. The reported uncertainty of these measurements was $\pm 10 \%$.

\section{Discussion of Results}

\section{A. Silica}

The solid conduction model developed by Lee and Cunnington $^{6}$ for Q-fiber was used in the present study. The measured effective thermal conductivity of Q-fiber insulation at densities of $48.6,68.8$, and $95.6 \mathrm{~kg} / \mathrm{m}^{3}$ at 0.001 torr was used to estimate the specific extinction coefficient. At this low pressure, gas conduction is negligible, and radiation and solid conduction are the only significant modes of heat transfer. The low-pressure experimental effective thermal conductivity data as a function of $\mathrm{T}_{\mathrm{H}}$ for the three sample densities is shown in Fig. 2. The symbols represent the measured data and the error bars represent the experimental uncertainties obtained from detailed uncertainty analysis for each data point. Average $T_{C}$ for these measurements was 293 $\pm 10 \mathrm{~K}$. The parameter estimation was accomplished by minimizing the sum of the square of differences between measured and predicted effective thermal conductivities. The predicted effective thermal conductivities were obtained by using various estimates of unknown parameters for the specific extinction coefficient to calculate the radiant thermal conductivity based on Eq. (5a), calculating the thermal conductivity according to Eq. (4), and then calculating the effective thermal conductivity based on Eq. (14) using the experimentally measured $\mathrm{T}_{\mathrm{H}}$ and $\mathrm{T}_{\mathrm{C}}$ as the integration limits. The variation of the experimentally estimated specific extinction coefficient with temperature for this insulation is shown in Fig. 3 along with the theoretical results for fibers randomly oriented in space from Lee and Cunnington. ${ }^{6}$ There is close agreement between the experimentally estimated and theoretical data with rms deviation of $4.8 \%$ over the temperature range of $400-1500 \mathrm{~K}$ (maximum deviation of $6.2 \%$ ), with the estimated 
values slightly over-predicting the theoretical values at all temperatures. The estimated maximum uncertainty in the theoretical data is $4 \%$, based on observed deviations from literature data for the imaginary part of the complex index of refraction of high purity vitreous silica with small diameter fibers. ${ }^{6}$ Given the uncertainties in both experimental and theoretical data, the close agreement between the theoretical and experimentally estimated specific extinction coefficients validates the experimental data and methodology used for estimating radiant properties from the experimental data. The experimentally estimated specific extinction coefficient variation for this insulation was then used to calculate the predicted effective thermal conductivity variation at 0.001 torr for the three sample densities as shown in Fig. 2 using dashed lines. Also shown in the figure are the calculated effective thermal conductivities using the theoretical specific extinction coefficient data plotted using solid lines. The rms deviation in calculated effective thermal conductivity using the theoretical and experimentally estimated specific extinction coefficient was $3.8 \%$, with maximum deviation of $4.8 \%$.

The contribution of gas conduction at higher pressures in nitrogen calculated from Eq. (8) was added to the radiation and solid conduction terms in Eq. (4), then integrated using Eq. (14) to obtain predicted effective thermal conductivities at higher pressures. The variation of measured and predicted effective thermal conductivities with $\mathrm{T}_{\mathrm{H}}$ for the sample density of 68.8 $\mathrm{kg} / \mathrm{m}^{3}$ at pressures of $0.1,1,10$, and 750 torr is shown in Fig. 4. The rms deviation between measurements and predictions for all test pressures and temperatures for this sample was $4.6 \%$, with rms deviations of $2.4,3.9,4.9,4.3$, 5.3 , and $2.9 \%$ at pressures of $0.01,0.1,1,10,100$, and 750 torr, respectively. The close agreement between measurements and predictions at various pressures validates the gas conduction model. Therefore, it can be concluded that the overall heat transfer model for Q-Fiber is validated over the density range of 48 to $96 \mathrm{~kg} / \mathrm{m}^{3}$ and over the temperature and pressure range of 300 to $1400 \mathrm{~K}$ and 0.001 to 760 torr, respectively. The validated model can then be used to calculate thermal conductivity as a function of temperature and pressure in any gaseous environment at any density between 48 and $96 \mathrm{~kg} / \mathrm{m}^{3}$. The calculated thermal conductivity of Q-Fiber at a density of $48 \mathrm{~kg} / \mathrm{m}^{3}$ as a function of temperature for pressures of $0.001,1,10,50$, and 750 torr in air is shown in Figs. 5.

\section{B. Alumina}

The solid conduction component of heat transfer for Saffil was estimated from the transient step heating technique thermal conductivity measurements on a sample at a density of $63 \mathrm{~kg} / \mathrm{m}^{3}$ at temperatures between 180 and $300 \mathrm{~K}$ at 0.005 torr in nitrogen. At these lower temperatures the radiation component of heat transfer is minimal, therefore, providing the highest sensitivity for estimation of parameters needed for solid conduction modeling. The symbols in Fig. 6 represent the thermal conductivity measurements, which consist of contributions of both solid conduction and radiation. This experimental data was used to infer the relevant

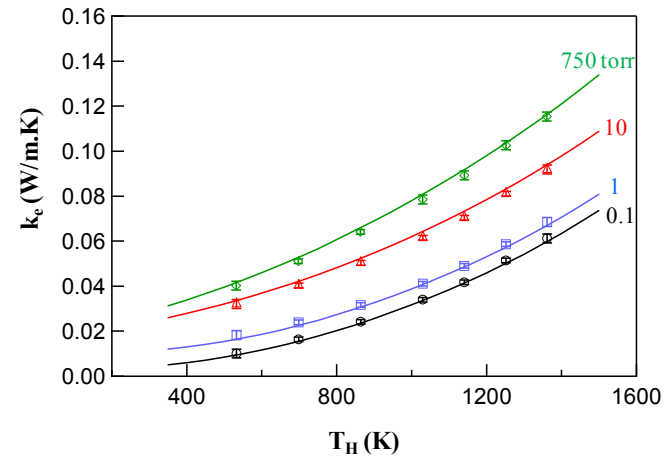

Figure 4. Comparison of measured and predicted effective thermal conductivities of $Q$ Fiber as a function of $T_{H}$ at a density of 68.8 $\mathrm{kg} / \mathrm{m}^{3}$ at various pressures in nitrogen.

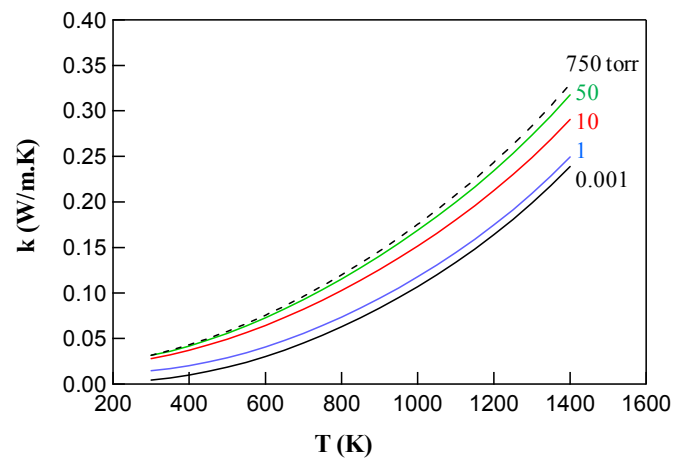

Figure 5. Predicted thermal conductivity of QFiber as a function of temperature at a density of $48 \mathrm{~kg} / \mathrm{m}^{3}$ at various pressures in air.

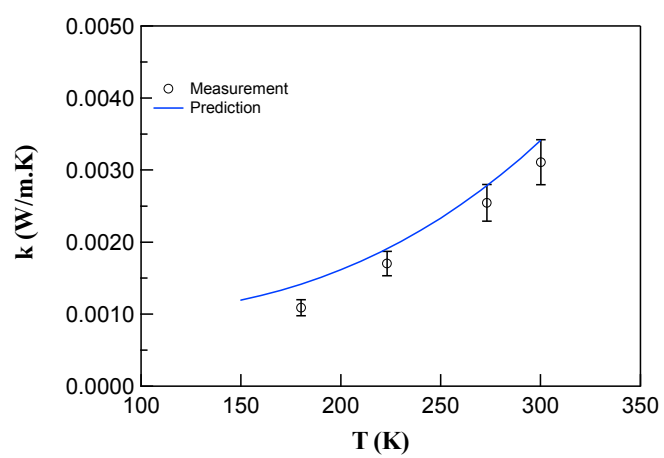

Figure 6. Predicted and measured thermal conductivity of Saffil at a density of $63 \mathrm{~kg} / \mathrm{m}^{3}$ at cryogenic temperatures at 0.005 torr in nitrogen. 
parameters for solid conduction, $F_{s}$ and $b$ in Eq. (7), with the resulting best-fit thermal model shown as the solid line in the figure. The ratio of solid thermal conductivity to total thermal conductivity, at this pressure and insulation density, varies from 0.6 to 0.2 between 180 and $300 \mathrm{~K}$, and from 0.09 to 0.001 between 400 and $1500 \mathrm{~K}$. Therefore, solid conduction is not a major contributor to overall heat transfer in this insulation for high temperature applications. The same is true for all the other highporosity insulations considered in this study.

Saffil effective thermal conductivity measurements at a density of $24 \mathrm{~kg} / \mathrm{m}^{3}$ at 0.001 torr pressure, shown as symbols in Fig. 7a, were used for estimating the specific extinction coefficient. The best estimate of variation of specific extinction coefficient with temperature for Saffil, shown in Fig. 8, resulted in the best fit of effective thermal conductivity shown as the solid line in Fig 7a. Results of theoretical modeling of the Saffil specific extinction coefficient using literature data for the complex refractive index of pure alumina departed significantly from the estimated values. As Saffil contains 4 to 5 weight percent of silica and its considerably lower density than pure alumina indicates there is probably microporosity in the structure which may have a strong effect on refractive index. To evaluate how well the radiation and conduction components of heat transfer have been modeled, the comparison of measured and predicted effective thermal conductivities at sample densities of 48,72 and $96 \mathrm{~kg} / \mathrm{m}^{3}$ at 0.001 torr pressure as a function of $\mathrm{T}_{\mathrm{H}}$ is shown in Fig. 7b. The rms deviations between measured and predicted effective thermal conductivities were $2.6,8.3$, and $1.5 \%$, for data at sample densities of 48,72 and $96 \mathrm{~kg} / \mathrm{m}^{3}$, respectively. These rms deviations were typically within the experimental uncertainties. The close agreement between predictions and measurements at 0.001 torr indicates that the radiation and solid conduction components of heat transfer through this alumina fibrous insulation at various densities has been modeled satisfactorily.

Comparisons of predicted and measured effective thermal conductivities at pressures of $0.5,2.5,5$, and 100 torr in nitrogen for a sample density of $48 \mathrm{~kg} / \mathrm{m}^{3}$ as a function of $\mathrm{T}_{\mathrm{H}}$ is shown in Fig. 9. The rms deviations between measured and predicted effective thermal conductivities were $5.9,3.8,4.5$, and $8.2 \%$ at pressures of $0.5,2.5,5$ and 100 torr, respectively, with predictions typically matching measurements to within the experimental uncertainties. The rms deviations between measurements and predictions for data over all pressures and temperatures for sample densities of 24, 48, 72, and 96 $\mathrm{kg} / \mathrm{m}^{3}$ were $6.1,6,8$, and $4.4 \%$, respectively, with an overall $\mathrm{rms}$ deviation of $6.4 \%$ for data at all densities.

The heat transfer model for Saffil was then compared to the transient step heating technique thermal conductivity measurements using a sample at a density of $144 \mathrm{~kg} / \mathrm{m}^{3}$. Comparison of predicted and measured thermal conductivities as a function of temperature for pressures of $0.005,1$, and 760 torr in nitrogen is shown in Fig. 10 . The predictions matched the experimental data within the experimental uncertainty range, with $10 \% \mathrm{rms}$ deviation between measurements and predictions, validating the heat transfer model. The specific extinction coefficient 


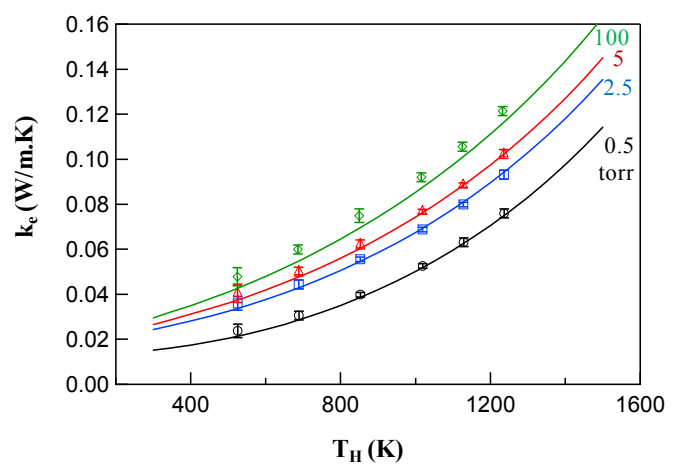

Figure 9. Comparison of measured and predicted effective thermal conductivities of Saffil as a function of $T_{H}$ at a density of 48 $\mathrm{kg} / \mathrm{m}^{3}$ at various pressures in nitrogen.

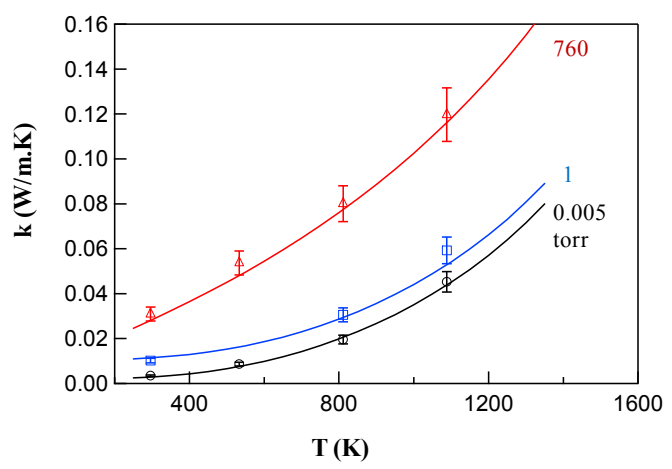

Figure 10. Variation of predicted and measured thermal conductivity of Saffil with temperature at a density of $144 \mathrm{~kg} / \mathrm{m}^{3}$ at various pressures in nitrogen.

estimated based on data at $24 \mathrm{~kg} / \mathrm{m}^{3}$ was found to produce accurate results for samples with densities between 48 and $144 \mathrm{~kg} / \mathrm{m}^{3}$. The validated heat transfer model can then be used to generate thermal conductivity predictions as a function of temperature and pressure in various gaseous media at various densities. Variation of thermal conductivity with temperature at various pressures for Saffil at densities of 48 and $96 \mathrm{~kg} / \mathrm{m}^{3}$ in air is shown in Figs. 11a and $11 \mathrm{~b}$, respectively.

It was assumed that the solid conduction model developed for Saffil was also applicable to APA. Effective thermal conductivity measurements of APA as a function of $\mathrm{T}_{\mathrm{H}}$ at 0.001 torr pressure for three different tests, shown in Fig. 12, was used to obtain the best estimate of the variation of specific extinction coefficient with temperature for this insulation, as shown in Fig. 8. The resulting predicted effective thermal conductivity for APA using this experimentally estimated specific extinction coefficient is also shown in Fig. 12 as the solid line. The three tests consisted of one test on one sample (Test 1) and two repeat tests on a different sample (Tests 2 and 3). The error bars shown in Fig. 12 are the detailed experimental uncertainties for one of the tests. As can be seen from the figure the experimental data are very repeatable. The average $T_{C}$ for the data shown in the figure was $294 \pm 7.6 \mathrm{~K}$. Even though the fiber material and fiber diameter for Saffil and APA are nominally the same, their specific extinction coefficients are slightly different, as seen in Fig. 8, because of differences in fiber orientations for the two insulations. For one of the tests on APA, embedded thermocouples were used at the center of the test sample at non-dimensional heights of $0.22,0.43$, 0.65 , and 0.87 , with non-dimensional heights of 0 and 1 corresponding to cold and hot-side boundaries. The effective thermal conductivity at each one of these locations was calculated using the in-depth temperatures and their

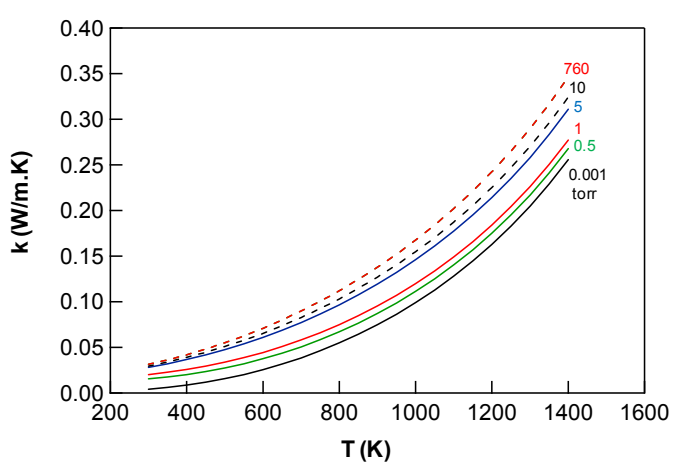

a)

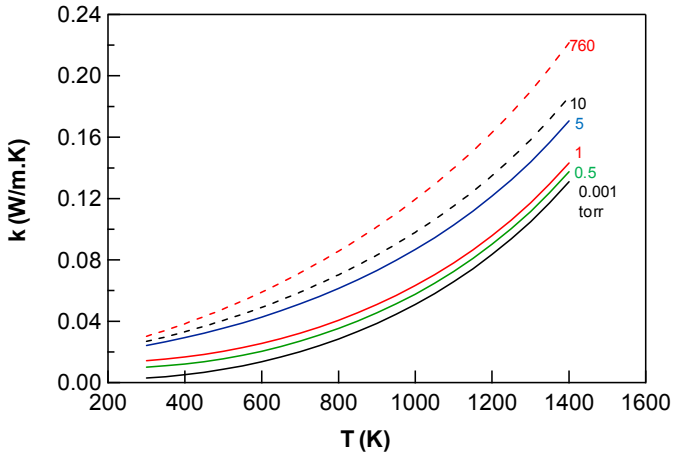

b)

Figure 11. Variation of predicted thermal conductivity of Saffil with temperature at various pressures in air at densities of: a) 48 $\mathrm{kg} / \mathrm{m}^{3}$, b) $96 \mathrm{~kg} / \mathrm{m}^{3}$. corresponding heights instead of $\mathrm{T}_{\mathrm{H}}$ and $\mathrm{L}$ from Eq. (13).

The variation of these in-depth local effective thermal conductivities with $T_{H}$ is shown in Fig. 13, along with the routine effective thermal conductivity measurements $(\mathrm{y} / \mathrm{L}=1)$, and predicted effective thermal conductivities. The local effective thermal conductivities from embedded thermocouples closely match the predicted effective thermal conductivities derived based on hot-side temperatures. The in-depth local effective thermal conductivity data 


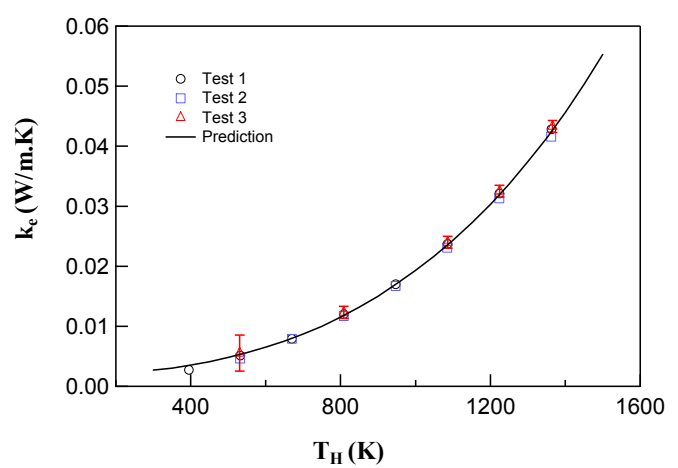

Figure 12. Predicted and measured effective thermal conductivities of APA as a function of $T_{H}$ at 0.001 torr in nitrogen.

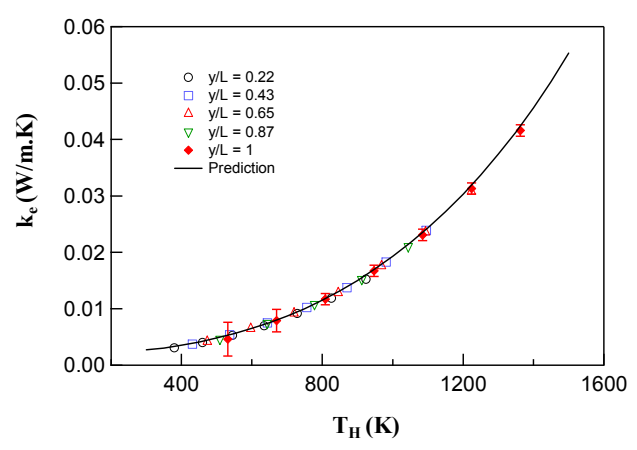

Figure 13. Comparison of predicted and measured effective thermal conductivities of APA from in-depth thermocouples at 0.001 torr in nitrogen.

generated from embedded thermocouples could have also been used for specific extinction coefficient estimation, providing a higher number of data points for the parameter estimation. It was decided to only use this data to evaluate the quality of the standard procedure for determining specific extinction coefficient solely based on hot side temperatures. The predicted steady-state temperature distributions as a function of non-dimensional height through the sample for tests at six different $\mathrm{T}_{\mathrm{H}}$ at 0.001 torr pressure are shown in Fig. 14, along with the measured temperatures. The thermocouple at the non-dimensional height of 0.87 failed for the two highest temperature tests. Overall, there is excellent agreement between measured and predicted in-depth temperatures.

The heat transfer model for APA was then compared to the transient step heating technique thermal conductivity measurements. Comparison of predicted and measured thermal conductivities as a function temperature at a pressure of 0.005 torr in nitrogen is shown in Fig. 15. The predictions matched the experimental data well within the experimental uncertainty range of $\pm 10 \%$, with rms deviation of $6.4 \%$ between measured and predicted thermal conductivities, thus validating the APA heat transfer model. Using this validated model, thermal conductivity data for this insulation can be calculated at various temperatures and pressures in any gaseous medium. The variation of thermal conductivity of APA with temperature at various pressures in nitrogen and argon are provided in Figs. $16 \mathrm{a}$ and $16 \mathrm{~b}$, respectively.

So far the validity of the overall methodology for estimating specific extinction coefficients from the experimental data has been established by the data presented on Q-Fiber, Saffil, and APA. The same validated methodology was then applied to the remaining insulations, ZYF and OFI, to be presented in the following sections. It should also be noted that the methodology used here could have also been used in conjunction with standard thermal conductivity data (with small temperature differences maintained across sample thickness) instead of the effective thermal conductivity data with large temperature differences.

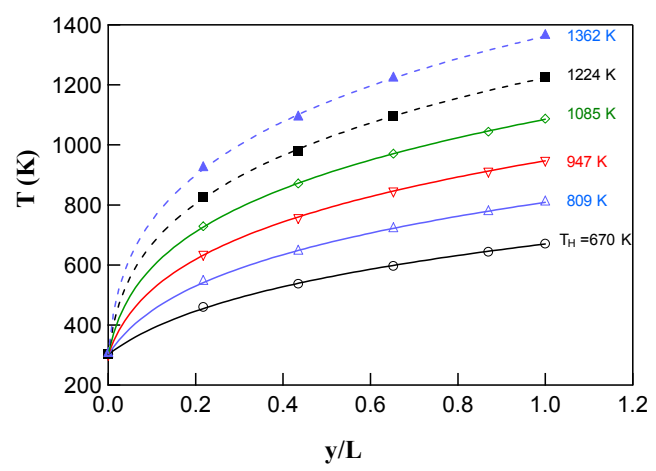

Figure 14. Comparison of predicted and measured in-depth temperatures in APA at various test temperatures at 0.001 torr in nitrogen.

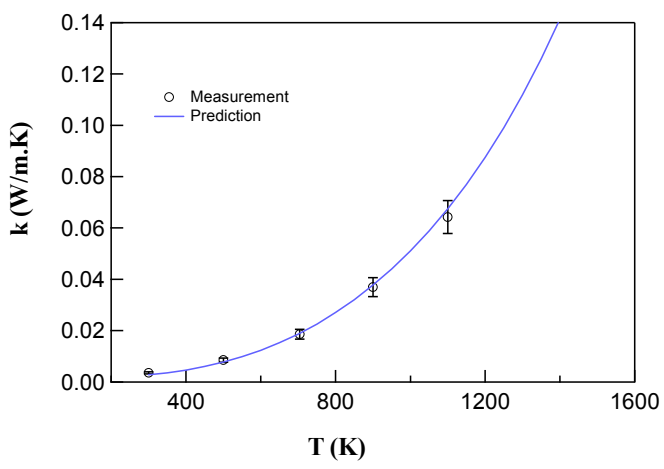

Figure 15. Variation of predicted and measured thermal conductivity of APA with temperature at 0.005 torr in nitrogen. 


\section{Zirconia}

The semi-empirical solid conduction model for ZYF fibrous insulation was estimated using the same methodology as was used for the silica and alumina insulations based on cryogenic measurements at 0.005 torr pressure. Since the effective index of refraction of this insulation was unknown, the combined parameter $\mathrm{e} / \mathrm{n}^{* 2}$ was estimated for this insulation. The measured effective thermal conductivity measurements of ZYF as a function of $T_{H}$ at 0.001 torr pressure are shown in Fig. 17 as symbols. This data was used to infer the best estimate of e $/ \mathrm{n}^{* 2}$ for this insulation, shown in Fig. 18. The resulting predicted effective thermal conductivities using this specific extinction coefficient is shown in Fig. 17 as the solid line. Embedded thermocouples were also used at the center of this test sample at non-dimensional heights of $0.09,0.27,0.45$, and 0.64 , with nondimensional heights of 0 and 1 corresponding to cold and hotside boundaries. The variation of in-depth local effective thermal conductivities with temperature is shown in Fig. 19, along with the routine effective thermal conductivity measurements $(y / L=1)$, and predicted effective thermal conductivities. The local effective thermal conductivities from embedded thermocouples closely match the predicted effective thermal conductivities derived based on hot-side temperatures. The predicted steady-state temperature distributions as a function of non-dimensional height through the sample for tests at seven different $T_{H}$ at 0.001 torr pressure are shown in Fig. 20, along with the measured temperatures. Overall, there is excellent agreement between measured and predicted in-depth temperatures. The variation of predicted thermal conductivity of ZYF with temperature in air at pressures of $0.001,1,5,10,50$, and 760 torr is shown in Fig. 21.

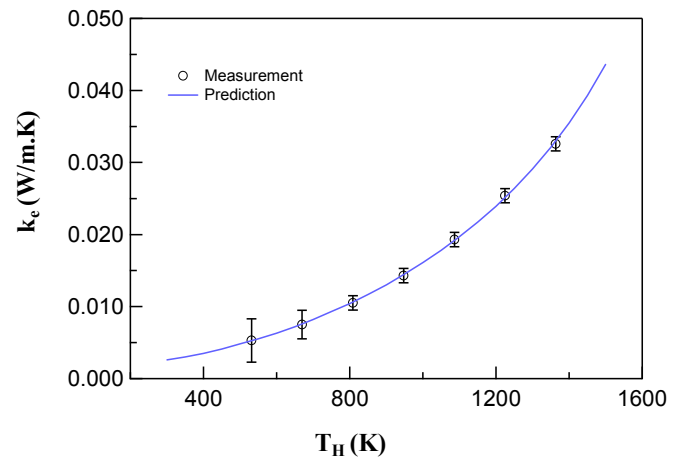

Figure 17. Predicted and measured effective thermal conductivities of ZYF as a function of $T_{H}$ at 0.001 torr in nitrogen.

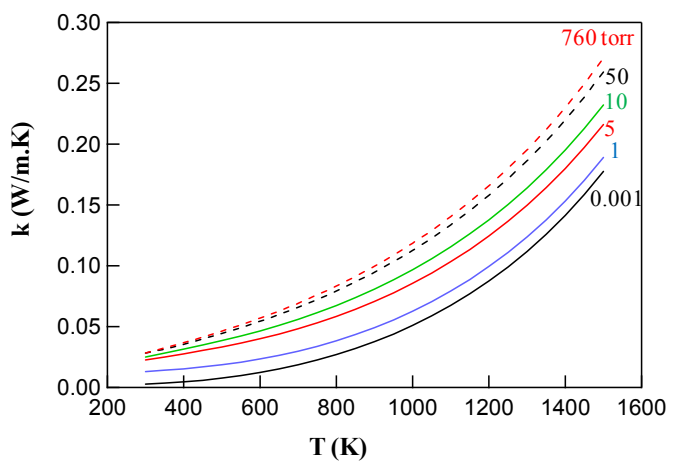

a)

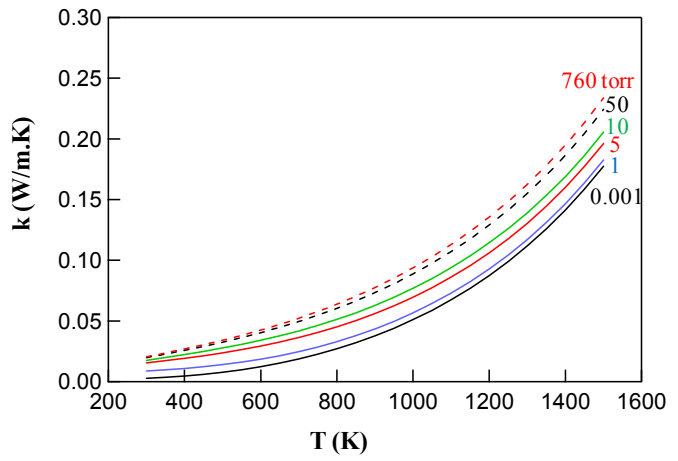

b)

Figure 16. Variation of predicted thermal conductivity of APA with temperature at various pressures in: a) nitrogen, b) argon.

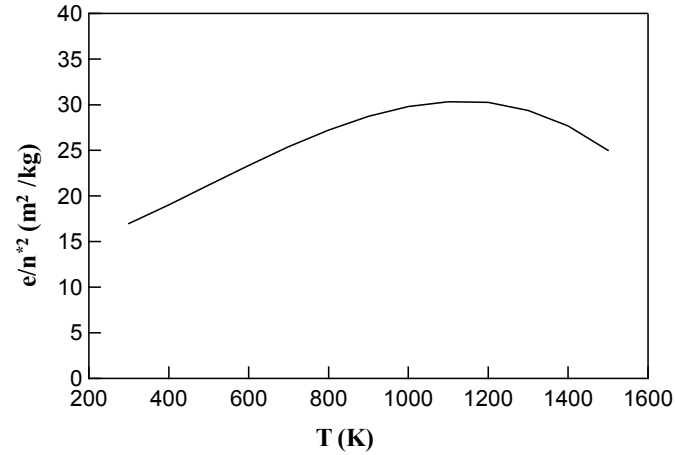

Figure 18. Experimentally estimated variation of $e / n^{* 2}$ with temperature for ZYF. 


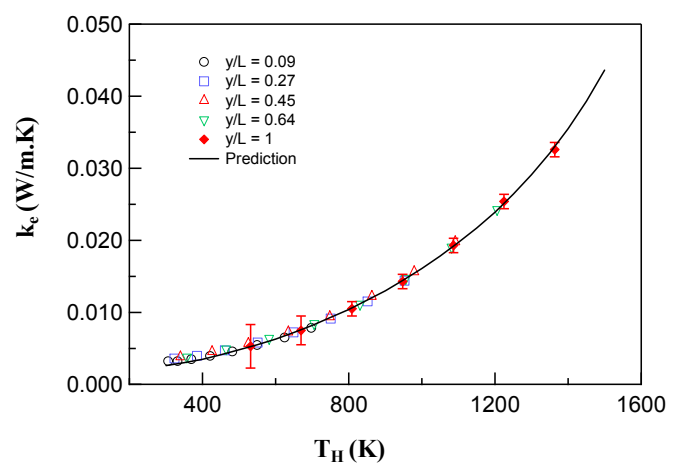

Figure 19. Comparison of predicted and measured effective thermal conductivities of ZYF from in-depth thermocouples at 0.001 torr in nitrogen.

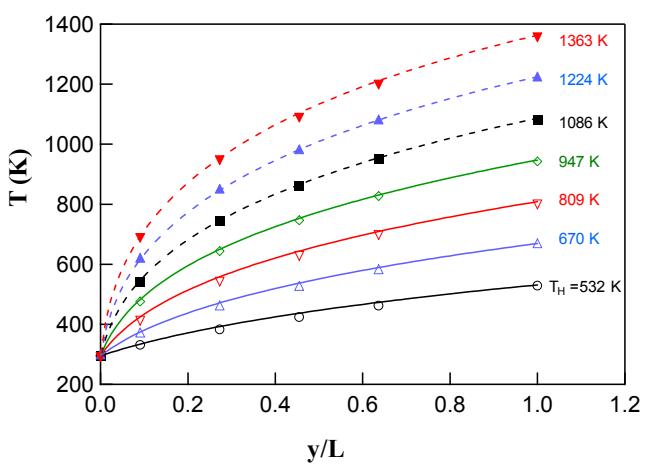

Figure 20. Comparison of predicted and measured in-depth temperatures in ZYF at various test temperatures at 0.001 torr in nitrogen.

\section{OFI}

It was assumed that the ceramic opacifiers embedded in the fibrous insulation mat would not have any effect on the solid conduction mode of heat transfer in this insulation. Therefore, the solid conduction modeling of Saffil was used for modeling this insulation. Since the radiant properties of Saffil have already been determined, the only unknown for this insulation was the specific extinction coefficient of opacifier. Effective thermal conductivity data were generated on this sample at 0.001 torr, shown as symbols in Fig. 22, and this data was then used to estimate best fit of specific extinction coefficient for the opacifier. The resulting predicted effective thermal conductivity data is also shown in Fig. 22 as a solid line. Despite large experimental uncertainties at the lower temperatures, the best fit predictions closely matched the experimental data. To evaluate the effectiveness of the opacifier in reducing the thermal conductivity as a result of attenuation of the radiation mode of heat transfer, comparison of thermal conductivity of OFI and Saffil at an effective density of $202.4 \mathrm{~kg} / \mathrm{m}^{3}$ as a function of temperature at 0.001 torr in air is provided in Fig. 23. It was assumed that Saffil could be manufactured at this higher density, and that the Saffil heat transfer model, validated over the density range of 24 to $140 \mathrm{~kg} / \mathrm{m}^{3}$, was applicable to this hypothetically higher density sample. The thermal conductivity of this OFI variation is on average $45 \%$ lower than Saffil at the same equivalent density. Therefore, the opacifers are more effective in reducing the radiation mode of heat transfer than simply increasing the density of Saffil. Comparison of OFI and ZYF at an effective density of $267 \mathrm{~kg} / \mathrm{m}^{3}$ as a function of temperature at 0.001 torr in air is provided in Fig. 24. The thermal conductivity of this OFI variation is on average $97 \%$ lower than ZYF at the same equivalent density. Therefore, it can be concluded that the use of efficient opacifiers is highly effective in reducing the radiation component of heat transfer in fibrous insulations.

Validated heat transfer models have been developed for various fibrous insulation samples at various densities. The product of thermal conductivity and density provides a good comparative quantity for comparing the steady-

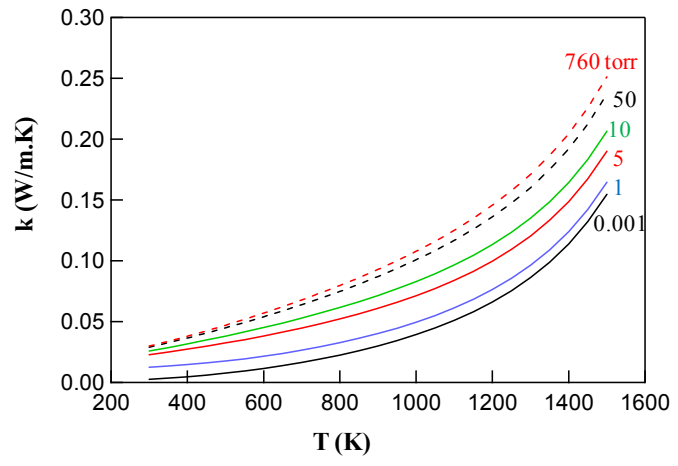

Figure 21. Variation of predicted thermal conductivity of ZYF with temperature at various pressures in air.

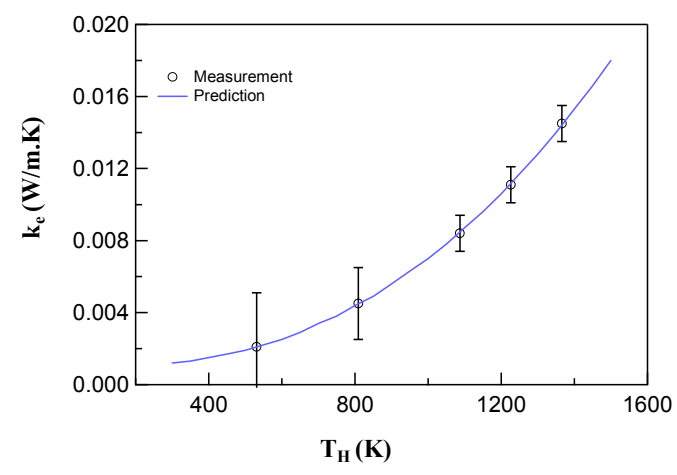

Figure 22. Predicted and measured effective thermal conductivities of OFI as a function of $T_{H}$ at 0.001 torr in nitrogen. 
state effectiveness of various samples. For steady-state heat transfer the product of thermal conductivity and density has been shown to be proportional to the mass of insulation required per unit area. ${ }^{25}$ The product of thermal conductivity and density of the various samples studied here as a function of temperature at a static pressure of 0.001 torr is shown in Fig. 25. The data presented consist of Q-Fiber, Saffil, APA, ZYF, and OFI at densities of 96, $96,107,267$, and $202.4 \mathrm{~kg} / \mathrm{m}^{3}$, respectively. OFI has the lowest $\rho k$, therefore, requiring less mass per unit area for a specific application. Q-Fiber and Saffil have similar performance. APA performance is similar to Saffil and Q-Fiber up to $1000 \mathrm{~K}$, but has slightly worse performance at higher temperatures. ZYF has the highest $\rho k$ over the entire temperature range, but has much higher temperature capability. The long duration exposure upper temperature limit for Q-Fiber, Saffil, APA, ZYF, and OFI are 1370, 1760, 1760, 2200, and $1600 \mathrm{~K}$, respectively.

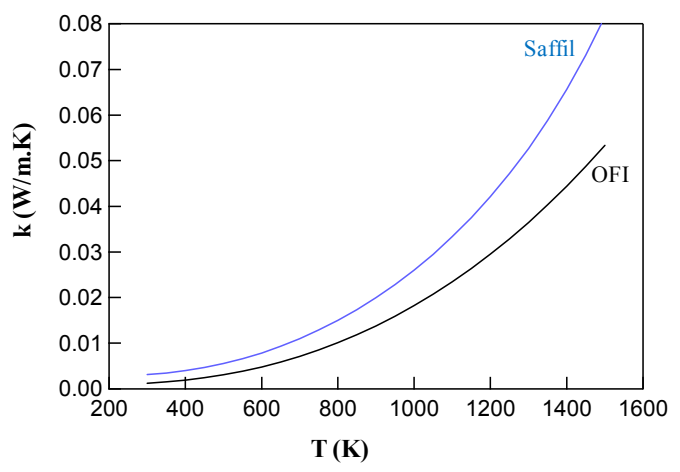

Figure 23. Comparison of predicted thermal conductivity of OFI and Saffil at a density of $202.4 \mathrm{~kg} / \mathrm{m}^{3}$ at 0.001 torr in air.

\section{Concluding Remarks}

Validated combined radiation and conduction heat transfer models were developed for five unbonded fibrous insulations. The specific extinction coefficients for the optically thick insulations were estimated from steady-state thermal test data with a large temperature gradient across the sample thickness at 0.001 torr static pressure. The steady-state test setup was found to provide accurate, repeatable test data. The solid conduction phase of heat transfer for these insulations was determined using semiempirical formulations in conjunction with standard thermal conductivity measurements in a vacuum at cryogenic temperatures. The experimentally estimated specific extinction coefficient for Q-Fiber was in good agreement with previously published theoretical data. The predicted thermal conductivity of Saffil and APA agreed well with standard thermal conductivity measurements using the transient step heating method. Once the radiation and solid conduction contributions are known from low pressure thermal data at 0.001 torr, the contribution of gas conduction at higher pressures for any gaseous medium can easily be calculated. It was shown that for low-pressure applications OFI was the most efficient insulation with the lowest product of density and thermal conductivity.

\section{Acknowledgments}

The authors wish to express their gratitude to following individuals for their invaluable contributions: Mr. Wayne Geouge, NASA Langley Research Center, for fabrication and instrumentation of steady-state test setup; Jozef Gembarovic, TPRL Inc, for providing transient step heating technique thermal conductivity measurements. This work was funded through NASA Fundamental Aeronautics Hypersonic Program, and the authors would like to 
express their gratitude to Dr. Anthony Calomino, NASA Glenn Research Center, for supporting this effort. Initial development of OFI had been funded by DARPA.

\section{References}

\footnotetext{
${ }^{1}$ Stark, C., and Fricke, J., "Improved Heat-Transfer Models for Fibrous Insulations," International Journal of Heat and Mass Transfer, Vol. 36, No. 3, 1993, pp. 617-625.

2 Daryabeigi, K., "Heat Transfer in High-Temperature Fibrous Insulation," Journal of Thermophysics and Heat Transfer, Vol. 17, No. 1, pp. 10-20, 2003.

${ }^{3}$ Banas, R. P., and Cunnington, G. R., "Determination of Effective Thermal Conductivity for the Space Shuttle Orbiter's Reusable Surface Insulation (RSI),” AIAA Paper 74-730, July 1974.

${ }^{4}$ Williams, S. D., and Curry, D. M., "Prediction of Rigid Silica Based Insulation Conductivity," NASA TP-3276, January 1993.
}

${ }^{5}$ Verschoor, J. D., Greebler, P., and Manville, N. J., "Heat Transfer by Gas Conduction and Radiation in Fibrous Insulations," Transactions of the American Society of Mechanical Engineers, Vol. 74, No. 8, 1952, pp. 961-968.

${ }^{6}$ Lee, S. C., and Cunnington, G. R., "Conduction and Radiation Heat Transfer in High-Porosity Fiber Thermal Insulation," Journal of Thermophysics and Heat Transfer, Vol. 14, No. 2, 2000, pp. 121-136.

${ }^{7}$ Gebhart, B., Heat Conduction and Mass Diffusion, McGraw-Hill, New York, 1993, pp. 442-444.

${ }^{8}$ Lee, S. C., and Cunnington, G. R, "Theoretical Models for Radiative Transfer in Fibrous Media," Annual Review of Heat Transfer, Edited by C. L. Tien, Vol. 9, Begell House, New York, 1998, pp. 159-212.

9 Roux, J. A., and Smith, A. M., "Combined Conductive and Radiative Heat Transfer in an Absorbing and Scattering Medium," American Institute of Chemical Engineers andAmerican Society of Mechanical Engineers Heat Transfer Conference, Paper 77-HT-50, Aug. 1977.

${ }^{10}$ Matthews, L. K., Viskanta, R., and Incropera, F. P., "Combined Conduction and Radiation Heat Transfer in Porous Materials Heated by Intense Solar Radiation,” Journal of Solar Energy Engineering, Vol. 107, No. 1, 1985, pp. 29-34.

${ }^{11}$ Tong, T. W., and Tien, C. L., "Radiative Heat Transfer in Fibrous Insulations-Part I: Analytical Study," Journal of Heat Transfer, Vol. 105, No. 1, 1983, pp. 70-75.

${ }^{12}$ Tong, T. W., Yang, Q. S., and Tien, C. L., "Radiative Heat Transfer in Fibrous Insulations-Part II: Experimental Study," Journal of Heat Transfer, Vol. 105, No. 1, 1983, pp. 76-81.

${ }^{13}$ Lee, S. C., "Radiative Transfer through a Fibrous Medium: Allowance for Fiber Orientation," Journal of Quantitative Spectroscopy and Radiative Transfer, Vol. 36, No. 3, 1986, pp. 253-263.

${ }^{14}$ Lee, S. C., "Scattering Phase Function for Fibrous Media," International Journal of Heat and Mass Transfer, Vol. 33, No. 10, 1990, pp. 2183-2190.

${ }^{15}$ Caren, R. P., "Radiation Heat Transfer from a Metal to a Finely Divided Particulate Medium", Journal of Heat Transfer, Vol. 91, 1969, pp. 154-156.

${ }^{16}$ Haupt, R. L., and Haupt, S. E., Practical Genetic Algorithms, Wiley, New York, 1998.

${ }^{17}$ Pettyjohn, R. R., "Thermal Conductivity Measurements on a Fibrous Insulation Material," Proceedings of the Seventh Conference on Thermal Conductivity, NBS Special Publication 302, Edited by D. R. Flynn and B. A. Peavy Jr., U.S. Government Printing Office, Washington, DC, 1968, pp 729-736.

${ }^{18}$ Keller, K., Blumenberg, J., and Tomsik, J., "Fibre Orientation and the Conduction of Heat by a Gas Enclosed in Ceramic Layers," Zeitschrift fuer Flugwissenschaften und Weltraumforschung, Vol. 12, July-Aug. 1988, p. 258-260.

${ }^{19}$ Kaganer, M. G., Thermal Insulation in Cryogenic Engineering, Israel Program for Scientific Translations Press, Jerusalem, 1969, pp. 75-76.

${ }^{20}$ Daryabeigi, K., "Effective Thermal Conductivity of High Temperature Insulations for Reusable Launch Vehicles," NASA TM-1999-208972, February 1999.

${ }^{21}$ Daryabeigi, K., "Thermal Analysis and Design Optimization of Multilayer Insulation for Reentry Aerodynamic Heating," Journal of Spacecraft and Rockets, Vol. 39, No. 4, 2002, pp. 509-514.

${ }^{22}$ Daryabeigi, K., "Analysis and Testing of High Temperature Fibrous Insulation for Reusable Launch Vehicles," AIAA Paper 99-1044, January 1999.

${ }^{23}$ Coleman, H. W., and Steele, W. G., Experimentation and Uncertainty Analysis for Engineers, Wiley, New York, 1989.

${ }^{24}$ Gembarovic, J., and Taylor, R. E, "A Method for Thermal Diffusivity Determination of Thermal Insulators," International Journal of Thermophysics, Vol. 28, No. 6, 2007, pp. 2164-2175.

${ }^{25}$ Blosser, M. L., "Advanced Metallic Thermal Protection Systems for Reusable Launch Vehicles," Ph.D. Dissertation, Mechanical and Aerospace Engineering Dept., University of Virginia, Charlottesville, VA, May 2000. 